\title{
Targeted Next-Generation Sequencing Reveals Novel TTN Mutations Causing Recessive Distal Titinopathy
}

\section{Evila, Anni}

$2017-11$

Evila , A, Palmio , J , Vihola , A, Savarese , M , Tasca, G, Penttila , S , Lehtinen , S , Jonson, P H , De Bleecker , J , Rainer , P , Auer-Grumbach , M , Pouget , J , Salort-Campana , E , Vilchez , J J , Muelas , N , Olive , M , Hackman , P \& Udd , B 2017 , ' Targeted Next-Generation Sequencing Reveals Novel TTN Mutations Causing Recessive Distal Titinopathy ' , Molecular Neurobiology , vol. 54 , no. 9 , pp. 7212-7223 . https://doi.org/10.1007/s12035-016-02

http://hdl.handle.net/10138/253441

https://doi.org/10.1007/s12035-016-0242-3

publishedVersion

Downloaded from Helda, University of Helsinki institutional repository.

This is an electronic reprint of the original article.

This reprint may differ from the original in pagination and typographic detail.

Please cite the original version. 


\title{
Targeted Next-Generation Sequencing Reveals Novel TTN Mutations Causing Recessive Distal Titinopathy
}

\author{
Anni Evilä ${ }^{1}$ - Johanna Palmio ${ }^{2}$ - Anna Vihola ${ }^{1}$ - Marco Savarese ${ }^{1,3}$ - Giorgio Tasca ${ }^{4,5}$. \\ Sini Penttilä ${ }^{2}$ - Sara Lehtinen ${ }^{2}$ • Per Harald Jonson ${ }^{1}$ • Jan De Bleecker ${ }^{6}$ - Peter Rainer $^{7}$. \\ Michaela Auer-Grumbach ${ }^{8}$ • Jean Pouget ${ }^{9}$ - Emmanuelle Salort-Campana ${ }^{10}$. \\ Juan J. Vilchez ${ }^{10,11}$ • Nuria Muelas ${ }^{10,11}$ • Montse Olive ${ }^{12}$ - Peter Hackman ${ }^{1}$. \\ Bjarne Udd ${ }^{1,2,13}$
}

Received: 1 February 2016/Accepted: 18 October 2016 /Published online: 29 October 2016

(C) Springer Science+Business Media New York 2016

\begin{abstract}
Tibial muscular dystrophy (TMD) is the first described human titinopathy. It is a mild adult-onset slowly progressive myopathy causing weakness and atrophy in the anterior lower leg muscles. TMD is caused by mutations in the last two exons, Mex5 and Mex6, of the titin gene (TTN). The first reported TMD mutations were dominant, but the Finnish founder mutation FINmaj, an 11-bp insertion/deletion in Mex6, in homozygosity caused a completely different severe early-onset limb-girdle muscular dystrophy 2J (LGMD2J). Later, we reported that not all TMD mutations cause LGMD when homozygous or compound heterozygous with truncating mutation, but some of them rather cause a more severe TMD-like distal disease. We have now performed targeted
\end{abstract}

Electronic supplementary material The online version of this article (doi:10.1007/s12035-016-0242-3) contains supplementary material, which is available to authorized users.

Anni Evilä

anni.evila@helsinki.fi

$1 \quad$ Folkhälsan Institute of Genetics, Biomedicum Helsinki and Department of Medical Genetics, Haartman Institute, University of Helsinki, Haartmaninkatu 8, 00290 Helsinki, Finland

2 Neuromuscular Research Center, University of Tampere and Tampere University Hospital, Tampere, Finland

3 Telethon Institute of Genetics and Medicine, Pozzuoli, Italy

4 Institute of Neurology, Policlinico "A. Gemelli” Foundation University Hospital, Rome, Italy

5 Don Carlo Gnocchi ONLUS Foundation, Milan, Italy

6 Neuromuscular Reference Center and Neurology Department, Ghent University Hospital and St. Lucas General Hospital, University of Ghent, Ghent, Belgium next-generation sequencing of myopathy-related genes on seven families from Albania, Bosnia, Iran, Tunisia, Belgium, and Spain with juvenile or early adult onset recessive distal myopathy. Novel mutations in TTN Mex5, Mex6 and A-band exon 340 were identified in homozygosity or compound heterozygosity with a frameshift or nonsense mutation in TTN Ior A-band region. Family members having only one of these $T T N$ mutations were healthy. Our results add yet another entity to the list of distal myopathies: juvenile or early adult onset recessive distal titinopathy.

Keywords Distal myopathy $\cdot$ Titinopathy $\cdot$ Titin $\cdot$ TTN

7 Division of Cardiology, Medical University of Graz, Graz, Austria

8 Department of Orthopaedics, Medical University of Vienna, Vienna, Austria

9 Neuromuscular and ALS Reference Center, La Timone Hospital, Aix-Marseille University, FILNEMUS, Marseille, France

10 Department of Neurology, Hospital Universitari i Politècnic La Fe, Valencia, Spain

11 Centro de Investigación Biomédica en Red de Enfermedades Raras (CIBERER), Madrid, Spain

12 Institute of Neuropathology, Department of Pathology and Neuromuscular Unit, Department of Neurology, IDIBELL-Hospital de Bellvitge, Hospitalet de Llobregat, Barcelona, Spain

13 Neurology Department, Vaasa Central Hospital, Vaasa, Finland 


\section{Introduction}

Tibial muscular dystrophy (TMD, OMIM \#600334) is an autosomal dominant distal myopathy characterized by weakness and atrophy especially in the tibialis anterior muscles of the lower leg. Clinical symptoms usually begin at the age of 3555 years, and the disease is slowly progressive $[1,2]$. TMD is caused by mutations in the last two exons, Mex 5 and Mex6 (exons 362 and 363), of the titin gene (TTN). The first reported TMD mutation was a Finnish founder mutation, FINmaj, an 11-bp insertion/deletion in TTN Mex6 leading to an in-frame exchange of four amino acids in the last M10 domain of titin [3]. In homozygous state, FINmaj causes a completely different phenotype, i.e., limb-girdle muscular dystrophy $2 \mathrm{~J}$ (LGMD2J, OMIM \#608807), which is a severe childhood onset disease affecting all proximal muscles. The disease onset is in the first or second decade, and the disease progresses over the next 20 years to wheelchair confinement $[1,4,5]$.

Loss of protein interactions of C-terminal titin is a likely consequence of the TMD/LGMD2J mutations, but only a few of these interactions are known. Myospryn, obscurin, and obscurin-like 1 are known to interact with titin M10 domain, and in LGMD2J muscles, mislocalization of obscurin has been observed [6, 7]. The FINmaj mutation in the last M10 domain of titin induces aberrant proteolytic processing of titin C-terminus, leading to a cleavage of a larger part of the titin C-terminus, according to Western blot and immunofluorescence studies of LGMD2J muscles [3, 8]. Next to the M10 domain is a unique is7 region, encoded by the alternatively spliced second last exon 362 (Mex5). It contains the M-line binding site for the musclespecific protease calpain 3 (CAPN3) [9]. Loss of the CAPN3 binding site in the M-band titin due to the disease related cleavage leads to a secondary CAPN3 reduction. The pathological cleavage in the titin is4-is5 region is not caused by CAPN3 but depends on other proteases, likely the ubiquitous calpains [8].

In addition to the FINmaj mutation, several other missense and truncating mutations in TTN Mex5 and Mex6 causing TMD have been reported in different European populations [3, 10-13] (Table 1). The French Mex5 TMD mutation (c.107647delT, p.S35883Qfs*10) was first reported to be dominant [11], but later studies revealed that both the mother and the son in the family also had different TTN frameshift mutations in compound heterozygosity with the Mex 5 mutation, making it possible that this mutation is recessive [14]. The rest of the reported mutations are dominant mutations in Mex6, although the Iberian TMD mutation does not always show a full penetrance when heterozygous [14]. More severe or atypical phenotypes have been observed in $9 \%$ of the patients with heterozygous TMD mutation [15]. We previously reported some of these patients as having a second TTN mutation in compound heterozygosity with TMD mutation explaining their atypical phenotypes [14]. We also discovered that not all TMD mutations cause LGMD when homozygous, but instead some of them cause a more severe TMD phenotype with earlier onset and progression to include soleus muscle and proximal muscles [14]. Mutations located outside the M-line titin have not previously been associated with distal myopathy.

We now describe ten patients from seven distal myopathy families. We identified novel mutations in TTN exons 362 (Mex5), 363 (Mex6), and 340 (A-band) in compound heterozygosity with frameshift or nonsense mutation in I-band or Aband TTN. In addition, one Belgian family had the previously reported Belgian TMD mutation in compound heterozygosity with an A-band nonsense mutation, and one Spanish family had the previously reported Iberian TMD mutation in compound heterozygosity with an A-band frameshift mutation.

\section{Patients and Methods}

\section{Patients}

DNA samples of patients and healthy family members of distal myopathy families were referred by clinicians in different countries. Patients belonged to seven unrelated families from Albania with the proband living in Italy, Bosnia, Iran, Tunisia, Belgium, and two unrelated Spanish families (Fig. 1).

The study was approved by the local ethics committees, and samples were obtained according to the Helsinki declaration.

\section{Targeted Next-Generation Sequencing}

Targeted next-generation sequencing of myopathy-related genes was performed on patients: AII:2, BII:1, CII:1, DII:4, EII:1, FII:3, and GII:2. DNA enrichment and next-generation sequencing were performed at the Institute for Molecular Medicine Finland (FIMM). DNA libraries were prepared using NEBNext DNA Sample Prep Master Mix Set 1 Kit (New England BioLabs), and four-plex pooled barcoded DNA libraries were captured using a designed SeqCap EZ Choice Library (Roche NimbleGen), MyoCap gene panel [16]. Paired-end sequencing (100 bp) was performed using Illumina HiSeq 1500 with a sequencing depth of $100 \times$. Next-generation sequence data analysis was performed using a pipeline described earlier [16]. Patient EII:1 was sequenced using MotorPlex gene panel at Telethon Institute of Genetics and Medicine [17]. The sequenced genes are listed in Supplementary Table 1 and the identified rare variants in Supplementary Table 2.

\section{Variant Analysis}

Exonic and splice site variants (within 15-bp of splice junctions) were analyzed. Variants with allele frequency higher than $1 \%$ in 1000 Genomes or ESP6500 databases were filtered out. The Human Gene Mutation Database (HGMD Professional 
Table 1 Previously reported TTN mutations causing distal myopathy (RefSeq NM 001267550.1 and NP 001254479.1)

\begin{tabular}{lllll}
\hline Origin & Exon & DNA & Protein & Reference \\
\hline France & 362/Mex5 & c.107647delT & p.S35883Qfs*10 & {$[11]$} \\
Finland/FINmaj & 363/Mex6 & $\begin{array}{c}\text { c.107780-107790 delAAGTAACA } \\
\text { TGG insTGAAAGAAAAA }\end{array}$ & $\begin{array}{c}\text { p.35927-35930 } \\
\text { delinsVKEK }\end{array}$ & {$[3]$} \\
Italy & 363/Mex6 & c.107837A $>$ C & p.H35946P & {$[12]$} \\
Belgium & 363/Mex6 & c.107840T>A & p.I35947N & {$[10]$} \\
France & $363 /$ Mex6 & c.107867T>C & p.L35956P & {$[3]$} \\
Spain & 363/Mex6 & c.107889delA & p.K35963Nfs*9 & {$[11]$} \\
France & $363 / M e x 6$ & c.107890C $>$ T & p.Q35964* & {$[11]$} \\
Switzerland & 363/Mex6 & c.107892-107897 delAGATGG & p.35964-35966delinsH & {$[13]$} \\
\hline
\end{tabular}

2014.3) was used to detect previously reported pathogenic mutations.

\section{Sanger Sequencing}

Sanger sequencing was performed to confirm the findings and to assess the segregation of the mutation with the disease in the families. Primers were designed using Primer3 software and PCR was performed with DreamTaq ${ }^{\mathrm{TM}}$ DNA Polymerase (Thermo Fisher Scientific) according to standard protocol. PCR products were sequenced on ABI3730xl DNA Analyzer (Applied Biosystems), using the Big-Dye Terminator v3.1 kit and analyzed with Sequencher 5.0 software (Gene Codes Corporation).

\section{Genotyping}

Families A, B, and C were genotyped for microsatellite markers D2S1244, D2S148, D2S2173, D2S300, D2S385, D2S324, D2S2978, and D2S384 spanning a region of $4.3 \mathrm{Mb}$ around TTN. Fluorescently labeled PCR products were analyzed using ABI3730x1 DNA Analyzer and GeneMapper v4.0 software (Applied Biosystems).

\section{RNA Extraction and Reverse-Transcription PCR (RT-PCR)}

The consequences of the mutations were studied by cDNA sequencing. RNA was extracted from frozen muscle biopsies of patients BII:2 and CII:1 using Direct-zol ${ }^{\mathrm{TM}}$ RNA MiniPrep Kit (Zymo Research), and cDNA was synthesized with RevertAid H Minus Reverse Transcriptase (Thermo Fisher Scientific) following the instructions. Primers were designed with Primer3 software, and fragments were amplified with DreamTaq ${ }^{\mathrm{TM}}$ DNA Polymerase (Thermo Fisher Scientific) and sequenced.

\section{Western Blotting}

Western blotting of muscle biopsy samples was performed according to standard methods, as described earlier [11, 14]. Two different primary antibodies raised against titin M10, rabbit polyclonal antibody M10-1 [11], and mouse
Family A (Albanian)

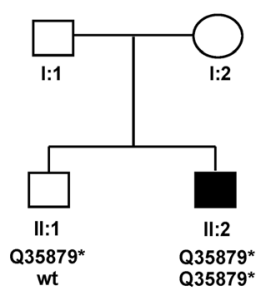

Family B (Bosnian)

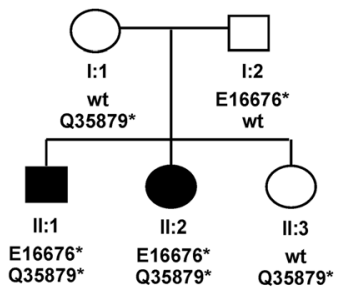

Family C (Iranian)

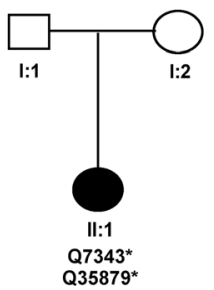

Family E (Belgian)

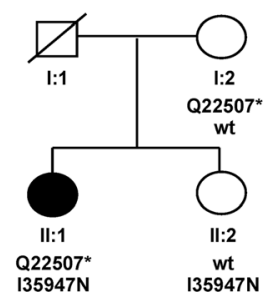

Family $F$ (Spanish)

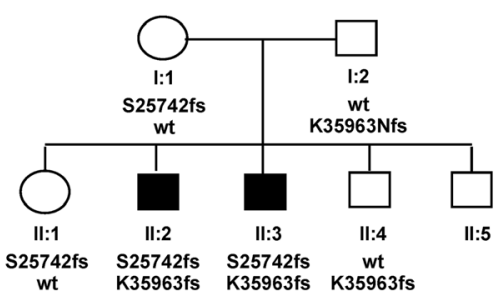

Fig. 1 Pedigrees of the families and identified TTN mutations in the individuals 
monoclonal antibody 11-4-3 [14] were used, followed by HRP-conjugated secondary antibodies (Dako, Glostrup, Denmark) and ECL detection using the Pierce SuperSignal West Femto substrate (Thermo Fisher Scientific).

\section{Results}

\section{Clinical Characteristics of the Patients}

The clinical details of each patient are shown in Table 2.

\section{Family A}

The proband was referred at age 36 when he underwent electrophysiological evaluation after an acute unilateral L4-L5 radiculopathy. Electromyography (EMG) showed fibrillation potentials on both tibialis anterior muscles, but the recruitment was myopathic. He had never complained of any problems before, apart from sciatica, except minor instability of the left ankle after a fracture 10 years before. Family history was unremarkable. On physical examination, he showed waddling gait and could not walk on heels. He had pes cavus and muscle atrophy in the left calf and quadriceps and bilaterally in the anterior compartment of the lower legs. There was mild postural tremor of the fingers but no weakness in the neck, scapular girdle, upper limb, or hand muscles. Creatine kinase (CK) level was mildly elevated. Muscle biopsy (right peroneus) showed myopathic changes without rimmed vacuolar pathology.

\section{Family $B$}

The proband (II:2) complained of unstable gait with frequent falls since age 25 years. On examination at age 41, there was prominent atrophy and weakness of the tibialis anterior muscles and she walked with steppage gait. Otherwise, there was no weakness or atrophy in proximal lower limb muscles, upper limbs, hands, or shoulder girdle. Patellar tendon reflexes were absent whereas Achilles reflexes were normal. She had no sensory disturbances. Electrophysiology showed nerve conduction within the normal range and slightly low amplitudes in the peroneal nerve. EMG of the tibialis anterior muscle revealed severe myopathic changes. CK level was slightly elevated. Cardiac dimensions and systolic and diastolic left ventricular function were normal on echocardiography, and the ECG was unremarkable. Cardiac troponin T and NTproBNP values were within normal limits.

Her brother (II:1) reported that he had always had a strange gait and had been unable to walk on heels. On examination at age 32, there was moderate weakness of toe and foot extensors and he walked with mild steppage gait. Patellar reflexes were absent and Achilles reflexes diminished. Sensory functions were normal. There was no weakness in the hands, but cardiomyopathy has been diagnosed years before and after an episode of acute tonsillitis and myocarditis. CK levels were mildly elevated.

One sister and the parents were normal on neurological examination with the exception of mild weakness of foot and toe extensors in the mother.

\section{Family $C$}

A 34-year-old female had noticed ankle instability and walking difficulties at age 29 . The symptoms slowly progressed to moderate foot drop. On examination she had marked atrophy in the calf muscles and in the anterolateral compartment of the leg more pronounced on the right. Ankle dorsiflexion weakness with steppage gait was evident, and mild weakness in thigh abduction was noted. There were mild contractures in the Achilles tendons. Otherwise, neurological examination was unremarkable. CK levels were slightly elevated. All three muscle biopsies performed showed unspecific myopathic changes and rimmed vacuolar pathology in one sample. The last biopsy taken from the left gastrocnemius medialis revealed a marked increase of internalized nuclei and core pathology. Immunohistochemistry showed accumulation of myotilin, alpha-B-crystallin, desmin, and p62/SQSTM1 in the core regions.

\section{Family D}

In family $\mathrm{D}$, two sisters were affected. Their father was Tunisian, and their mother was French of Italian and Hungarian descent. The proband (II:2) presented with waddling gait at age 24. Proximal and distal weakness progressed slowly. On physical examination at age 40 , she walked with waddling and steppage gait and was unable to get up from a squatting position. There was no weakness in the upper limbs, neck, or abdominal muscles, although she complained of difficulties in raising arms. She had bilateral atrophy in tibialis anterior muscles and mild atrophy in the right quadriceps. Weakness was evident in proximal lower limb and tibialis anterior muscles. Nerve conduction studies were within the normal range, but EMG showed a myopathic pattern in quadriceps and tibialis anterior muscles.

Her sister (II:4) complained of difficulties in walking down stairs at the age of 24 . On examination at age 38 , she walked with waddling gait and was unable to stand on heels. She had left quadriceps and bilateral tibialis anterior muscle atrophy. There was moderate weakness of iliopsoas and hamstrings muscles.

Both patients had absent ankle reflexes without any sensory disturbances and CK levels were slightly elevated. Muscle pathology (left peroneus) in both sisters showed variation in fiber size with sparse atrophic fibers and mild myofibrillar abnormalities such as moth-eaten fibers on oxidative stains. 


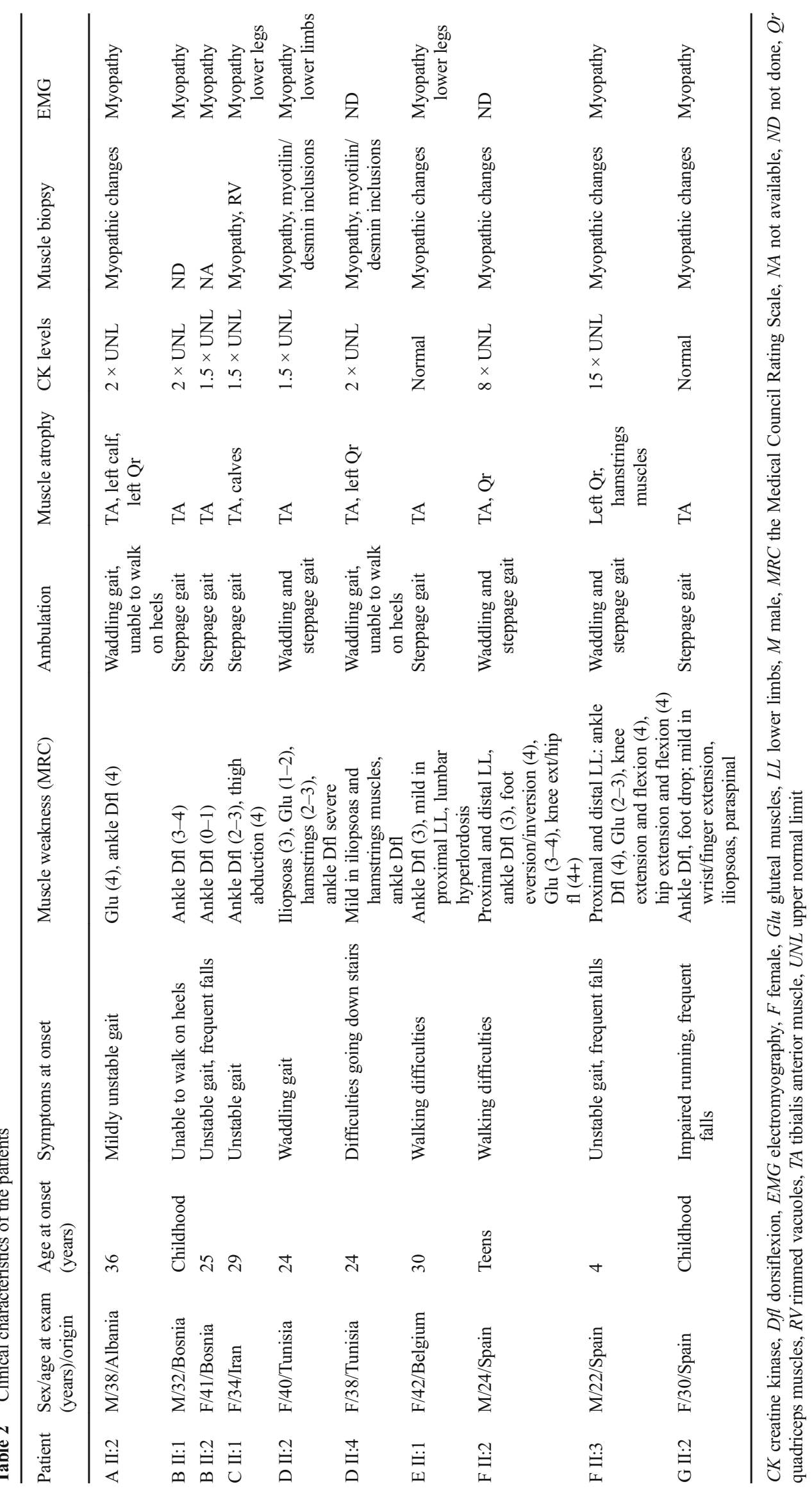


Sarcoplasmic and subsarcolemmal inclusions were strongly reactive for myotilin and desmin on immunohistochemistry.

\section{Family E}

The proband is a 42-year-old woman whose mother and sister were healthy, but her father had died of cardiac problems at the age of 40 . Since age 30 , the patient had a slowly deteriorating gait. She walked with steppage gait due to bilateral weakness in ankle dorsiflexion. There was atrophy of the anterior compartment of lower leg muscles, a bilateral slightly asymmetric Trendelenburg sign, mild weakness in quadriceps and hamstring muscles and lumbar hyperlordosis. Both Achilles tendons were shortened without foot deformities. No major abnormalities were present in the upper limbs or shoulder girdle. CK levels were at the upper normal limit, and EMG showed a myopathic pattern, above all in tibialis anterior muscles. Muscle biopsy (left quadriceps) evidenced myopathic changes with atrophic fibers (predominantly type I) and variation in fiber size. Immunohistochemistry studies were normal. No cardiomyopathy was detected on ultrasound.

\section{Family F}

Two brothers, aged 28 (II:2) and 26 years (II:3), were affected. The father was Spanish, and the mother was originally from Morocco. Motor milestones were normal in both. The proband (II:3) presented with frequent trips and falls and tendency to walk on toes from 4 years old. Later at school, he reported difficulties with climbing stairs and running. His brother (II:2) was referred at age 22 due to gait disturbances and tendency to stumble from teens. Both also reported exercise intolerance, myalgia, and cramps. Muscle weakness was predominant in toe and ankle extensors and hip abductors. Both had contractures in the Achilles tendons and one associated pes cavus. Atrophy was present in the proximal lower limbs and anterior leg muscles in II:2 and in pelvic-femoral muscles in II:3. They walked with hyperlordotic, waddling and steppage gait and were not able to stand on heels. CK levels were high (10-15 × normal limit), and the EMG showed a myopathic pattern. There was no evidence of cardiorespiratory involvement. Muscle biopsies (quadriceps and gastrocnemius) revealed myopathic features with normal immunohistochemistry studies but with calpain 3 reduction on Western blot.

\section{Family $G$}

The proband is a 37 -year-old woman with no family history. She presented during late childhood with mild muscle weakness, which caused difficulties in running and frequent falls. During her 20, she developed generalized muscle weakness predominantly involving distal and axial muscles. At the age of 29 she underwent surgery due to a small atrial septum defect. Examination revealed mild scoliosis, bilateral scapular winging, and bilateral ankle contractures. There was severe weakness in both tibialis anterior muscles with foot drop. She had generalized muscle slenderness and mild muscle weakness involving the neck flexors, humeral rotators, paraspinal muscles, wrist and finger extensors, as well as iliopsoas. CK levels were normal. Muscle biopsy (biceps brachii) showed myopathic changes, increased number of internal nuclei, some fibers with cytoplasmic bodies and several fibers containing minicores.

\section{Muscle Imaging Findings}

Muscle MRI was performed on at least one patient from each family (Fig. 2). Fatty degenerative changes were present in all patients. Their distribution was variable, but a common feature was severe degeneration of tibialis anterior in all but one patient (FII:3) with widespread weakness in the proximal lower limb muscles. Soleus and hamstrings were frequently affected, while gastrocnemius medialis and lateralis were usually spared. Almost all imaging studies showed asymmetrical involvement. At the pelvic level, gluteus minimus and medius muscles were affected in patient EII:1. In FII:2 and FII:3, there was also involvement of gluteus, predominantly in medius.

\section{Sequencing Results}

The targeted next-generation sequencing of myopathy-related genes revealed both novel (not present in 1000 Genomes or Exome Sequencing Project databases) and previously reported TTN mutations in the patients (Table 3 and Fig. 3). Three of the families, A, B, and $\mathrm{C}$, had a novel nonsense mutation c.107635C>T (p.Q35879*) in TTN Mex5 in either homozygous state or in compound heterozygosity with another nonsense mutation. Family D has a novel missense mutation c.107743A>C (p.T35915P) in TTN Mex6 in compound heterozygosity with a frameshift mutation. Family $\mathrm{E}$ had the previously reported Belgian TMD mutation c.107840T>A (p.I35947N) [10] in compound heterozygosity with a nonsense mutation and family $\mathrm{F}$ had the previously reported Iberian TMD mutation c.107889delA (p.K35963Nfs*9) [11] in compound heterozygosity with a frameshift mutation. Family $\mathrm{G}$ with a novel distal myopathy phenotype had a novel missense mutation c.94285T>A (p.W31429R) in TTN Aband exon 340 in compound heterozygosity with a nonsense mutation. Family members with only one of these TTN mutations were healthy and had not been examined for muscle weakness (Fig. 1).

Families A, B, and $\mathrm{C}$ with the novel nonsense mutation c.107635C >T (p.Q35879*) in TTN Mex5 were genotyped with microsatellite markers, and they shared the same 
Fig. 2 Muscle imaging findings of the seven families. a Patient AII:2. The left soleus and gastrocnemius lateralis are most severely affected while tibialis anterior muscles are moderately affected. Involved also are peroneus muscle on the left, hamstrings on the left, but only semitendinosus on the right. $\mathbf{b}$ Patient BII:1. Severe fatty degenerative changes are seen in both tibialis anterior muscles and partly in soleus muscle more on the left. c Patient CII:1. Fatty degenerative changes are present in the anterolateral compartment of the leg more on the right, bilaterally in soleus muscles and in gastrocnemius medialis on the right. Thigh muscles are unaffected. d Patient DII:2. Tibialis anterior, extensor digitorum longus and soleus muscles are bilaterally and severely degenerated. The changes are more pronounced on the left as also tibialis posterior and flexor dig longus muscles are affected, whereas, at the thigh level the changes are more severe on the right. e Patient EII:1. Severe fatty degenerative changes are seen bilaterally in tibialis anterior, extensor digitorum longus, and soleus muscles. At the thigh level, hamstrings are more affected on the left. $\mathbf{f}$ Patient FII:3. Moderate changes are seen in peroneus muscles and in soleus on the left. In the thigh level, hamstrings are more severely affected with asymmetrical involvement of vasti muscles. $\mathbf{g}$ Patient GII:2. Severe fatty degenerative changes are present in both tibialis anterior muscles, while early changes are seen in gastrocnemius medialis, and hamstrings on the left

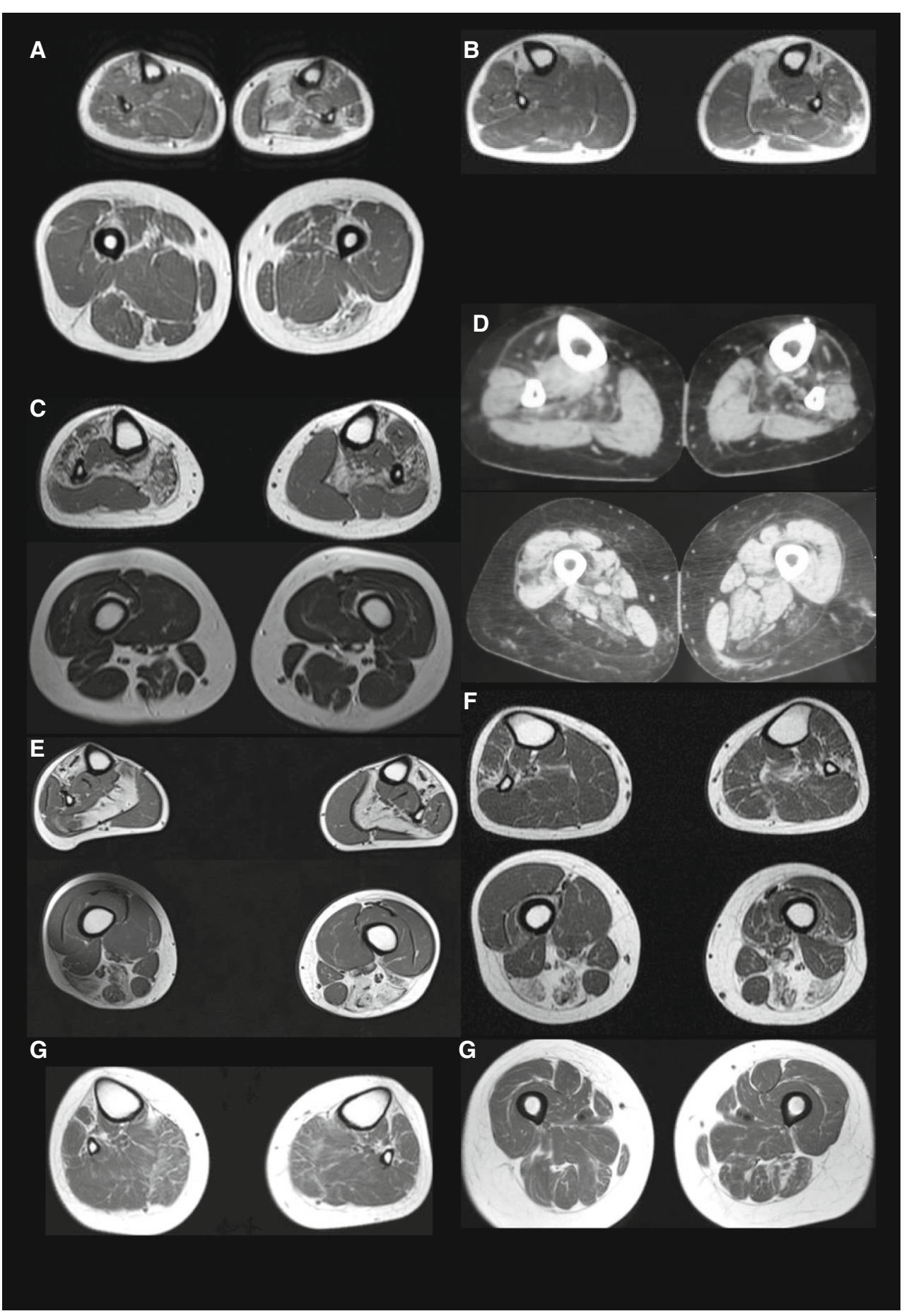

haplotype spanning from marker D2S148 to D2S324 around $T T N$, suggesting a common origin of the mutation (Fig. 4).

\section{Molecular Biological Findings}

The detected mutations were studied by RT-PCR from muscle biopsies of patients BII:2 and CII:1, which have the novel TMD mutation p.Q35879* in combination with a nonsense mutation. Regions spanning TTN exons 75-77, 265-267, and
361-363 were amplified from the cDNA and sequenced. Although RT-PCR per se is not a quantitative method, the sequencing chromatograms suggested unequal expression of the TTN alleles with lower signals from the allele having the nonsense mutation p.E16676* in BII:2 and p.Q7343* in CII:1 compared to the TTN allele containing the novel TMD mutation p.Q35879* (Fig. 5).

Titin protein was studied from muscle samples of patients AII: 2 and CII: 1 by Western blotting using antibodies for M10 
Table $3 T T N$ mutations identified in the patients (RefSeq NM 001267550.1 and NP $001254479.1)$

\begin{tabular}{|c|c|c|c|c|c|}
\hline Family & Origin & Exon & DNA & Protein & Reference \\
\hline A & Albania & 362 & c. $107635 \mathrm{C}>\mathrm{T}$ (homozygous) & p.Q35879* (homozygous) & \\
\hline \multirow[t]{2}{*}{ B } & Bosnia & 266 & c. $50026 \mathrm{G}>\mathrm{T}$ & p.E16676* & \\
\hline & & 362 & c. $107635 \mathrm{C}>\mathrm{T}$ & p.Q35879* & \\
\hline $\mathrm{C}$ & Iran & 76 & c. $22027 \mathrm{C}>\mathrm{T}$ & p.Q7343* & \\
\hline $\mathrm{D}$ & Tunisia & $\begin{array}{l}362 \\
326\end{array}$ & $\begin{array}{l}\text { c. } 107635 \mathrm{C}>\mathrm{T} \\
\text { c. } 85011 \_85014 \mathrm{del}\end{array}$ & $\begin{array}{l}\text { p.Q35879* } \\
\text { p.E28338Hfs*9 }\end{array}$ & \\
\hline \multirow[t]{2}{*}{$\mathrm{E}$} & Belgium & $\begin{array}{l}363 \\
319\end{array}$ & $\begin{array}{l}\text { c. } 107743 \mathrm{~A}>\mathrm{C} \\
\text { c. } 67519 \mathrm{C}>\mathrm{T}\end{array}$ & $\begin{array}{l}\text { p.T35915P } \\
\text { p.Q22507* }\end{array}$ & {$[10]$} \\
\hline & Spain & $\begin{array}{l}363 \\
326\end{array}$ & $\begin{array}{l}\text { c. } 107840 \mathrm{~T}>\mathrm{A} \\
\text { c. } 77226 \_77229 \mathrm{del}\end{array}$ & $\begin{array}{l}\text { p.I35947N } \\
\text { p.S25742Rfs*9 }\end{array}$ & [11] \\
\hline $\mathrm{F}$ & Spain & $\begin{array}{l}363 \\
306\end{array}$ & $\begin{array}{l}\text { c. } 107889 \mathrm{del} A \\
\text { c. } 63625 \mathrm{C}>\mathrm{T}\end{array}$ & $\begin{array}{l}\text { p.K35963Nfs*9 } \\
\text { p.R21209* }\end{array}$ & \\
\hline G & & 340 & c. $94285 \mathrm{~T}>\mathrm{A}$ & p.W31429R & \\
\hline
\end{tabular}

domain. Patient AII:2 was homozygous for the novel TMD mutation p.Q35879* and patient CII:1 had the same mutation in compound heterozygosity with a nonsense mutation p.Q7343* in exon 76. Reduction of C-terminal titin protein fragments was observed in both patient samples (Fig. 6).

\section{Discussion}

Novel recessive distal myopathy causing mutations in TTN exons 362 (Mex5), 363 (Mex6), and 340 (A-band) were identified in homozygous state or in compound heterozygosity together with I-band or A-band frameshift or nonsense mutations. The novel mutations in TTN Mex5 (c.107635C > T, p.Q35879*) and Mex6 (c.107743A $>$ C, p.T35915P) were located in the same exons as previously reported TMD mutations (Table 1). And they cause similar more severe distal titinopathy phenotypes as some of the previously reported Mex5/6 mutations (French p.S35883Qfs*10 and Iberian p.K35963Nfs*9) when they are compound heterozygous with truncating A-band mutations [14]. The novel missense Aband mutation (c.94285T>A, p.W31429R) in TTN exon 340 in the Spanish family $\mathrm{G}$ is the first identified TTN mutation in the A-band region to be associated with a distal myopathy. The muscle pathology usually showed minicore-core like changes that corresponded to protein aggregates or bodies when studied by myotilin, desmin, or alphaB-crystallin antibodies. In the samples tested, calpain 3 was reduced on Western blots, but characteristically no consistent rimmed vacuolar pathology was observed.

Affected members in three of the families, Albanian (A), Bosnian (B), and Iranian (C), had the same novel nonsense
A
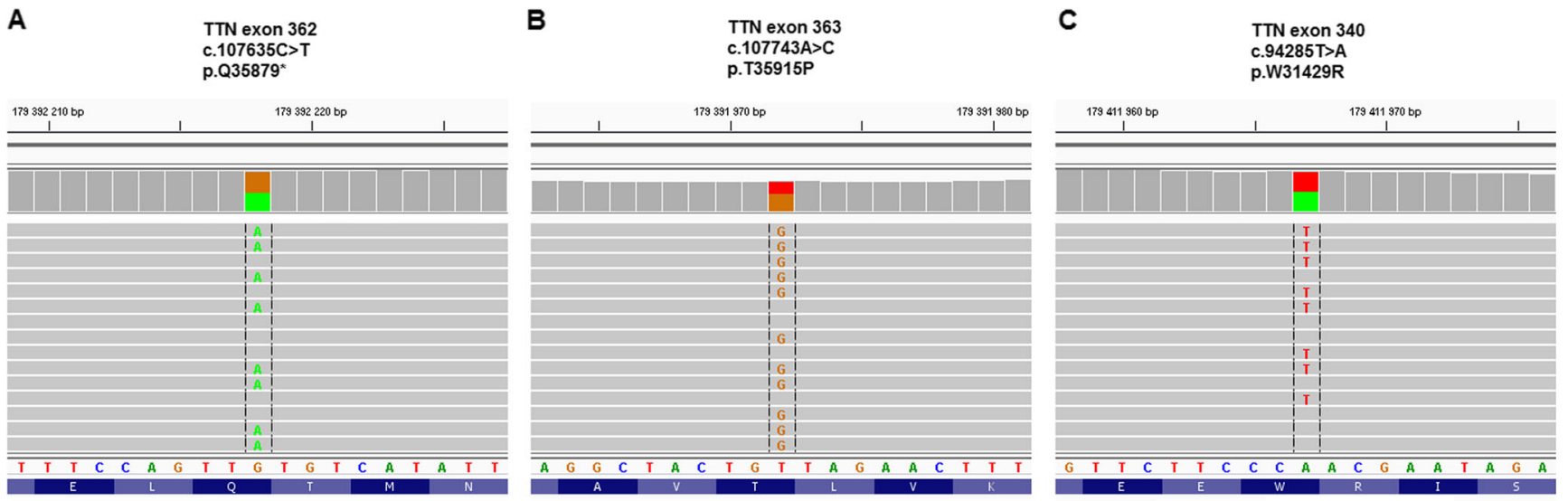

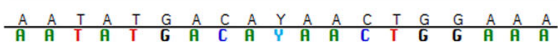
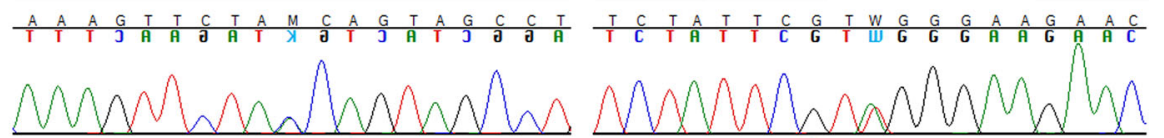

Fig. 3 Three novel recessive distal titinopathy mutations identified in the patients shown in Integrative Genomics Viewer (IGV) and Sequencher. a A novel nonsense mutation c. $107635 \mathrm{C}>\mathrm{T}$ (p.Q35879*) was identified in families A, B, and C. b A novel missense mutation c.107743A $>$ C
p.T35915P was identified in family D. c The first distal titinopathy mutation c.94285T>A (p.W31429R) in titin A-band was identified in family G 
Fig. 4 The Albanian (A), Bosnian (B), and Iranian (C) families with the novel nonsense mutation c. $107635 \mathrm{C}>\mathrm{T}$ (p.Q35879*) in TTN Mex5 shared the same haplotype spanning from marker D2S148 to D2S324 around TTN, which suggest a common origin of the mutation

\begin{tabular}{|c|c|c|c|c|c|c|c|c|c|c|c|c|c|c|c|c|c|}
\hline Marker & Position & $A: 1$ & $\mathrm{II}: 1$ & $A: I$ & $1: 2$ & B: & & B:I & $: 2$ & B:I & & B:I & & B:II & & & \\
\hline D2S1244 & 177238218 & 1 & 15 & 15 & 13 & 9 & 4 & & & 4 & 4 & 4 & 4 & 13 & 4 & 4 & 15 \\
\hline $2 S 148$ & 178231423 & 2 & 3 & 3 & 3 & 1 & 3 & 2 & 7 & 2 & 3 & 2 & 3 & 7 & $\checkmark$ & 2 & 3 \\
\hline 2 2S2173 & 1784 & 3 & 4 & 4 & 4 & 3 & 4 & 3 & 3 & 3 & 4 & 3 & 4 & 3 & 4 & 3 & 4 \\
\hline D2S300 & 1786 & 1 & 2 & 2 & 2 & 1 & 2 & 2 & 2 & 2 & 2 & 2 & 2 & 2 & 2 & 2 & 2 \\
\hline D2S385 & 1796 & 2 & 2 & 2 & 2 & 5 & 2 & 2 & 2 & 2 & 2 & 2 & 2 & 2 & 2 & 1 & 2 \\
\hline D2S324 & 1796 & 3 & 1 & 1 & 1 & 3 & 1 & 1 & 1 & 1 & 1 & 1 & 1 & 1 & 1 & 6 & \\
\hline D2S2978 & 1811 & 2 & 3 & 3 & 3 & 4 & T & 3 & 2 & 3 & 4 & 3 & 4 & 2 & 4 & 5 & 3 \\
\hline $2 S 384$ & 181502826 & 2 & 1 & 1 & 6 & 2 & 2 & 2 & 2 & 2 & 2 & 2 & 2 & 2 & & 2 & 2 \\
\hline
\end{tabular}

mutation c. $107635 \mathrm{C}>\mathrm{T}$ (p.Q35879*) in TTN Mex5 in either homozygous state or in compound heterozygosity with nonsense mutation in TTN I- or A-band region. They also shared the same haplotype around TTN suggesting a common origin of the mutation. Previously, only one Mex5 TMD mutation (c.107647delT, p.S35883Qfs*10) has been reported in a French family with two affected members. They were later discovered to have also TTN frameshift mutations in compound heterozygosity [14]. The French patients had similar more severe distal myopathy phenotype as the Albanian, Bosnian, and Iranian patients described here. The novel Mex 5 mutation is located 46 nucleotides upstream of the last exon-exon junction, which should spare the mRNA from nonsense-mediated decay (NMD), since NMD is thought to occur when the premature stop codon is located $>50-55$ nucleotides upstream of the last exon-exon junction [18]. In our RT-PCR results, the intensity of the I-band and A-band nonsense mutated alleles was consistently lower compared to the Mex 5 mutated allele (Fig. 5) and we hypothesized that this could reflect a lower expression due to NMD. Similar phenomenon has been reported previously [19, 20], but quantitative PCR would be needed to confirm it. Western blotting revealed clear reduction of M10-positive titin fragments, but not complete absence of all titin bands like in LGMD2J (Fig. 6). This is likely due to the presence of isoforms lacking is7, encoded by the alternatively spliced Mex5. These isoforms also lack the mutation and are normal. NMD is often incomplete, but the exact amount of translated truncated titin protein in the patients is not known. Nonsense and frameshift mutations in different parts of TTN have been suggested to
Fig. 5 cDNA sequencing chromatograms of patients BII:2 and CII:1 from mutated TTN regions. The chromatograms show lower signal from the exon 266 nonsense mutated (c.50026G>T, p.E16676*) allele in patient BII:2 (a) and exon 76 nonsense mutated (c. $22027 \mathrm{C}>\mathrm{T}$, p.Q7343*) allele in patient CII:1 (b) compared to the allele with the novel TMD mutation (c.107635C>T, p.Q35879*). In the novel TMD mutation region, higher signal is seen in the TMD allele compared to the other allele in both patients, BII: 2 (c) and CII:1 (d). The results suggest unequal expression of the TTN alleles with lower expression of the TTN alleles having nonsense mutations in exons 266 and 76 compared to the allele with novel TMD mutation in exon 362

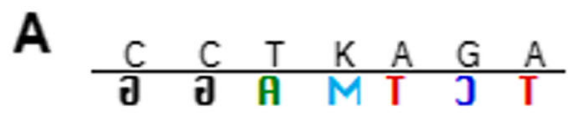

Bll:2

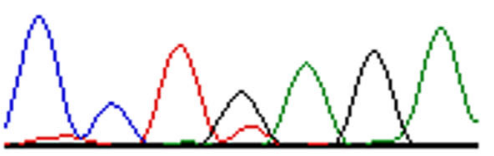

TTN exon 266

c. $50026 \mathrm{G}>\mathrm{T}$ p.E16676*

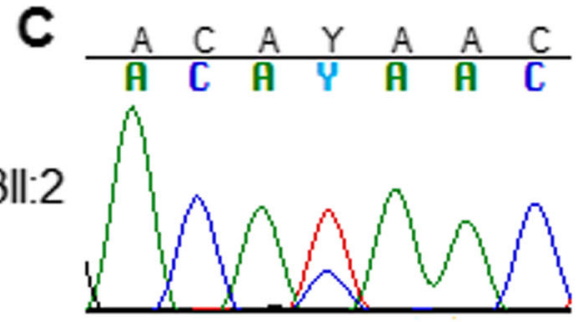

TTN exon 362

c. $107635 \mathrm{C}>\mathrm{T}$ p.Q35879*
B

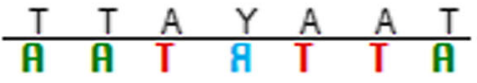

$\mathrm{Cll}: 1$

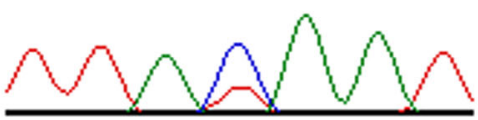

TTN exon 76

c. $22027 \mathrm{C}>\mathrm{T}$

p.Q7343*

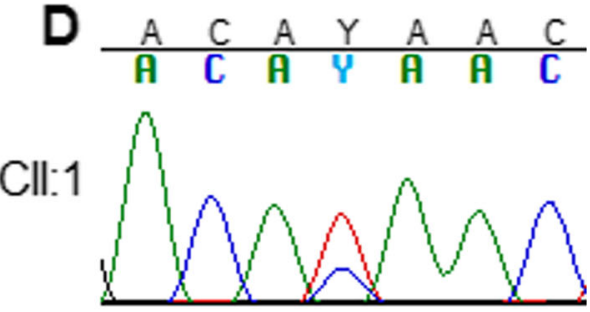

TTN exon 362

C. $107635 \mathrm{C}>\mathrm{T}$

p.Q35879* 
Fig. 6 Western blotting of skeletal muscle lysates with antibodies M10-1 and 11-4-3 shows a pattern of titin C-terminal fragments in healthy control, which are reduced in FINmaj heterozygous TMD, and virtually absent in FINmaj homozygous LGMD2J. In patients AII:2 and CII: 1 the bands are severely reduced, but not completely absent. Bands of $\sim 75$ and $\sim 28 \mathrm{kDa}$ represent unspecific cross-reactions. Myosin heavy chain (MyHC) was used as a loading control

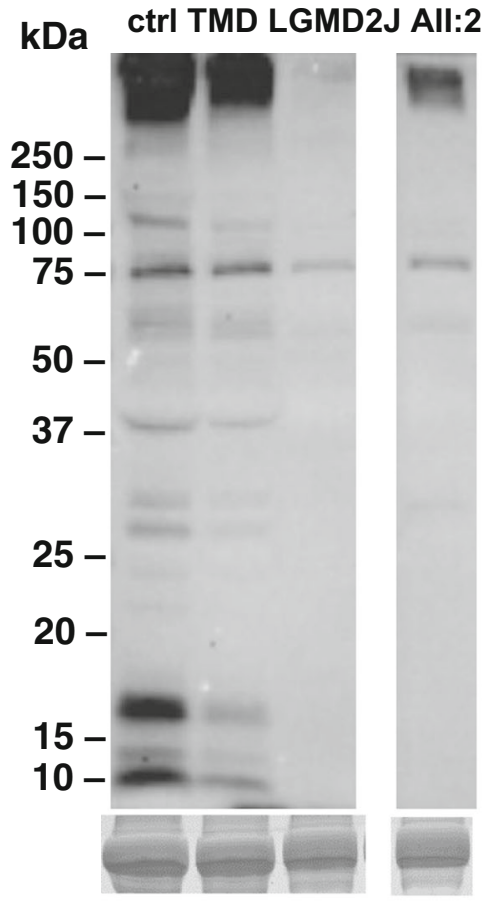

ctrl TMD LGMD2J CII:1

kDa

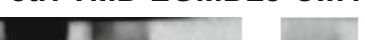

250

$150=$

$100-$

$75-$

$50-$

$37-$

$25-$

$20-$

$15-$

$10-$

\section{M10-1}

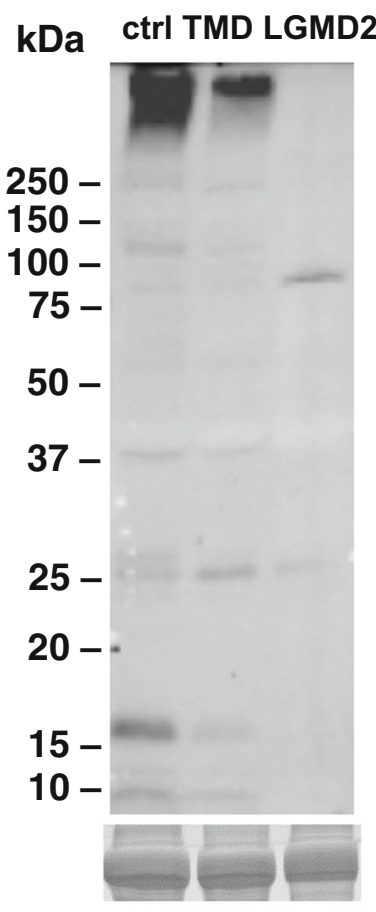

J All:2
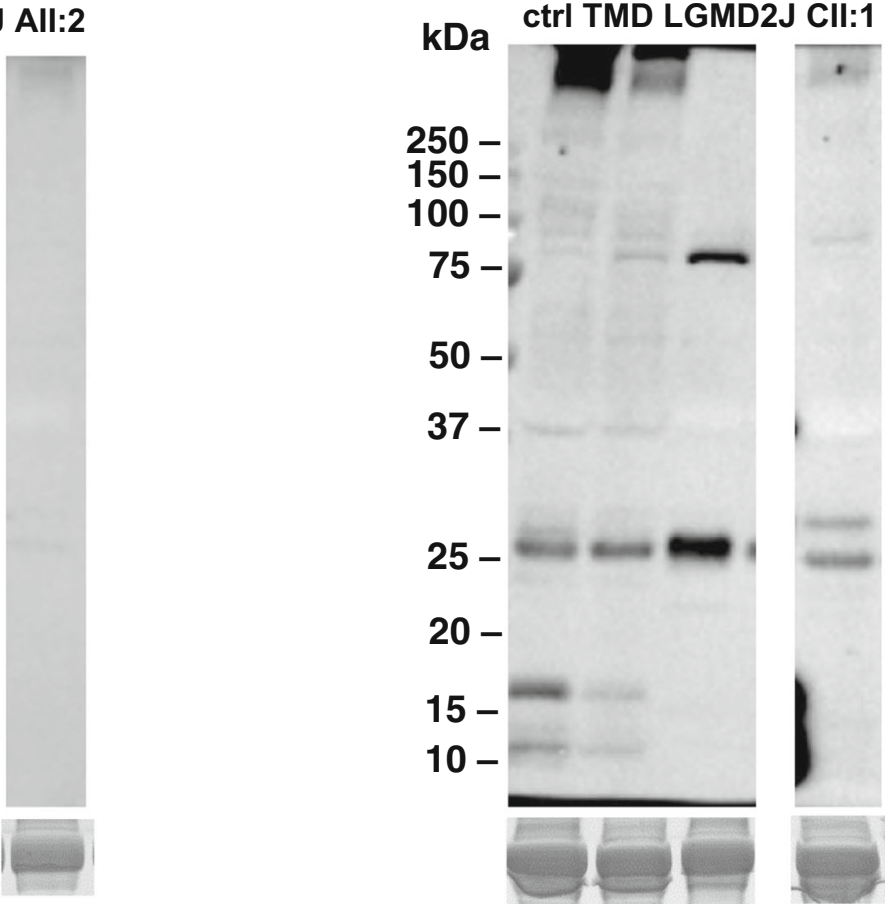

\section{1-4-3}

(Fig. 1). The Bosnian patient BII:1 had cardiomyopathy, which is likely caused by something else than TTN mutations, since the patient's sister with the same combination of TTN cause dominant cardiomyopathy [21], but this was not the case in the patients of our study, since family members carrying only one nonsense or frameshift mutation were healthy 
mutations did not have signs of cardiac disease on echocardiography, ECG, and laboratory examinations.

Affected members in the Tunisian family D had a novel missense mutation c.107743A $>$ C (p.T35915P) in TTN Mex6 in compound heterozygosity with an A-band frameshift mutation. The novel Mex6 mutation is located 12 amino acids upstream of the FINmaj TMD mutation and is thus the most N-terminal of the Mex6 mutations reported so far. The mutation was present in a heterozygous state in the healthy sibling DII:1, which indicates that the mutation does not cause a disease alone or it may cause a milder very late onset TMD disease, not detectable at age 49.

The affected member in the Belgian family $\mathrm{E}$ had the previously reported Belgian TMD mutation in compound heterozygosity with an A-band nonsense mutation and the affected members in the Spanish family F had the previously reported Iberian TMD mutation c.107889delA (p.K35963Nfs*9) in compound heterozygosity with an A-band frameshift mutation. The father in the Spanish family had only the Iberian TMD mutation and was healthy at age 57 . We have previously reported other individuals with heterozygous Iberian TMD mutation being healthy at age 55-60, indicating reduced penetrance or even recessive properties of the mutation [14]. Both the Belgian and the Iberian TMD mutations have been reported to cause very mild TMD phenotypes when heterozygote $[10,11]$. The Belgian TMD mutation affects a structurally less important amino acid residue compared to the FINmaj mutation that disrupts key structural features of the M10 Ig-fold. Yeast two-hybrid and GST-pulldown assays have shown that the FINmaj mutation reduces titin and obscurin/obscurin-like 1 binding severely, whereas binding by the Belgian TMD mutation is not detectably reduced [7].

The pathomechanism of TMD mutations has been studied with the FINmaj mutation, but this mutation differs from the other TMD mutations, since it causes an LGMD phenotype when homozygous or compound heterozygous with nonsense/frameshift mutation. Compound heterozygosities with truncating mutations are not known for most of the other TMD Mex6 mutations. The French Mex5 and Iberian Mex6 mutations cause a more severe TMD phenotype when they are compound heterozygous with truncating A-band mutations [14]. One patient with homozygous French Mex6 TMD mutation (c.107890C>T, p.Q35964*) has been described and he had proximal muscle weakness, but involvement of also distal muscles [22]. Reasons for variability in the phenotypes are currently not known. On heterozygous state, the reported TMD mutations cause similar late-onset mild distal myopathy and it is not excluded that the novel Mex5 and Mex6 mutations described here also could cause a very late onset mild TMD in heterozygosity.

A novel TTN A-band exon 340 mutation c.94285T>A (p.W31429R) was identified in compound heterozygosity with an A-band nonsense mutation in one patient in the
Spanish Family G. The mutation exchanges a conserved hydrophobic tryptophan, buried in an FN3 domain, to a positively charged arginine. It most probably breaks the hydrophobic core of the domain and leads to an unfolded FN3. Putative ligands for this domain are dysferlin and angiomotin [23]. The mutation most likely disrupts all the interactions of the domain. The patient had a novel phenotype with childhood onset and severe weakness in anterior compartment of legs combined with milder weakness in wrists, fingers and shoulders.

We now describe late childhood, juvenile or early adult onset recessive distal titinopathy caused by mutations in TTN Mex 5 and Mex6 either in homozygosity or compound heterozygosity with a truncating mutation in TTN I- or A-band region. Moreover, we describe the first distal titinopathy $\mathrm{mu}-$ tation in TTN A-band, which combined with a truncating Aband mutation, causes a phenotype with symptoms also in upper limbs, adding yet another entity to the list of distal myopathies. The exact molecular pathogenesis leading to differential muscle involvement with different TTN mutations is not clarified, although differential gene and isoform expression in muscles may constitute one underlying factor [24].

Acknowledgments This study was supported by the Folkhälsan Research Foundation, the Jane and Aatos Erkko Foundation, Association Française contre les Myopathies (SB/CP2013-0106, B.U.), the Academy of Finland (no. 138491, B.U.), the Sigrid Jusélius Foundation, the Liv och Hälsa Foundation, The Finnish Cultural Foundation (A.E.), Austrian Science Fund (FWF, P27634FW, M.A.), Tampere University Hospital Research Funds (B.U.), Fondo de Investigaciones Sanitarias-Instituto de Salud Carlos III (PI14-00738, M.O.), and the European Regional Development Fund (FEDER) (M.O.).

\section{Compliance with Ethical Standards}

Conflict of Interest The authors declare that they have no conflict of interest.

\section{References}

1. Udd B, Kääriäinen H, Somer H (1991) Muscular dystrophy with separate clinical phenotypes in a large family. Muscle Nerve 14: 1050-1058

2. Udd B, Partanen J, Halonen P, Falck B, Hakamies L, Heikkilä H, Ingo S, Kalimo H et al (1993) Tibial muscular dystrophy. Late adult-onset distal myopathy in 66 Finnish patients. Arch Neurol 50:604-608

3. Hackman P, Vihola A, Haravuori H, Marchand S, Sarparanta J, De Seze J, Labeit S, Witt C et al (2002) Tibial muscular dystrophy is a titinopathy caused by mutations in TTN, the gene encoding the giant skeletal-muscle protein titin. Am J Hum Genet 71:492-500

4. Udd B (1992) Limb-girdle type muscular dystrophy in a large family with distal myopathy: homozygous manifestation of a dominant gene? J Med Genet 29:383-389

5. Udd B, Rapola J, Nokelainen P, Arikawa E, Somer H (1992) Nonvacuolar myopathy in a large family with both late adult onset 
distal myopathy and severe proximal muscular dystrophy. J Neurol Sci 113:214-221

6. Sarparanta J, Blandin G, Charton K, Vihola A, Marchand S, Milic A, Hackman P, Ehler E et al (2010) Interactions with M-band titin and calpain 3 link myospryn (CMYA5) to tibial and limb-girdle muscular dystrophies. J Biol Chem 285:30304-30315

7. Fukuzawa A, Lange S, Holt M, Vihola A, Carmignac V, Ferreiro A, Udd B, Gautel M (2008) Interactions with titin and myomesin target obscurin and obscurin-like 1 to the M-band: implications for hereditary myopathies. J Cell Sci 121:1841-1851

8. Charton K, Sarparanta J, Vihola A, Milic A, Jonson PH, Suel L, Luque H, Boumela I et al (2015) CAPN3-mediated processing of C-terminal titin replaced by pathological cleavage in titinopathy. Hum Mol Genet 24:3718-3731

9. Sorimachi H, Kinbara K, Kimura S, Takahashi M, Ishiura S, Sasagawa N, Sorimachi N, Shimada H et al (1995) Musclespecific calpain, p94, responsible for limb girdle muscular dystrophy type $2 \mathrm{~A}$, associates with connectin through IS2, a p94-specific sequence. J Biol Chem 270:31158-31162

10. Van den Bergh PY, Bouquiaux O, Verellen C, Marchand S, Richard I, Hackman P, Udd B (2003) Tibial muscular dystrophy in a Belgian family. Ann Neurol 54:248-251

11. Hackman P, Marchand S, Sarparanta J, Vihola A, Penisson-Besnier I, Eymard B, Pardal-Fernandez JM, Hammouda E et al (2008) Truncating mutations in C-terminal titin may cause more severe tibial muscular dystrophy (TMD). Neuromuscul Disord 18:922928

12. Pollazzon M, Suominen T, Penttilä S, Malandrini A, Carluccio MA, Mondelli M, Marozza A, Federico A et al (2010) The first Italian family with tibial muscular dystrophy caused by a novel titin mutation. J Neurol 257:575-579

13. Suominen T, Udd B, Hackman P (2012) Udd distal myopathy. In: Pagon RA et al (eds) GeneReviews. University of Washington, Seattle, Seattle Updated 08/23/2012, accessed 06/11/2103. Available at: http://www.ncbi.nlm.nih.gov/books/NBK1323/;

14. Evilä A, Vihola A, Sarparanta J, Raheem O, Palmio J, Sandell S, Eymard B, Illa I et al (2014) Atypical phenotypes in titinopathies explained by second titin mutations. Ann Neurol 75:230-240
15. Udd B, Vihola A, Sarparanta J, Richard I, Hackman P (2005) Titinopathies and extension of the M-line mutation phenotype beyond distal myopathy and LGMD2J. Neurology 64:636-642

16. Evilä A, Arumilli M, Udd B, Hackman P (2015) Targeted nextgeneration sequencing assay for detection of mutations in primary myopathies. Neuromuscul, Disord

17. Savarese M., Di Fruscio G., Mutarelli M., Torella A., Magri F., Santorelli F. M., Comi G. P., Bruno C. et al., (2014) MotorPlex provides accurate variant detection across large muscle genes both in single myopathic patients and in pools of DNA samples. Acta Neuropathol Commun 2:100-014-0100-3

18. Maquat LE (2004) Nonsense-mediated mRNA decay: splicing, translation and mRNP dynamics. Nat Rev Mol Cell Biol 5:89-99

19. Renbaum P, Kellerman E, Jaron R, Geiger D, Segel R, Lee M, King MC, Levy-Lahad E (2009) Spinal muscular atrophy with pontocerebellar hypoplasia is caused by a mutation in the VRK1 gene. Am J Hum Genet 85:281-289

20. Hsu AP, Johnson KD, Falcone EL, Sanalkumar R, Sanchez L, Hickstein DD, Cuellar-Rodriguez J, Lemieux JE et al (2013) GATA2 haploinsufficiency caused by mutations in a conserved intronic element leads to MonoMAC syndrome. Blood 121:3830$7 \mathrm{~S} 1-7$

21. Herman DS, Lam L, Taylor MR, Wang L, Teekakirikul P, Christodoulou D, Conner L, DePalma SR et al (2012) Truncations of titin causing dilated cardiomyopathy. N Engl J Med 366:619-628

22. Penisson-Besnier I, Hackman P, Suominen T, Sarparanta J, Huovinen S, Richard-Cremieux I, Udd B (2010) Myopathies caused by homozygous titin mutations: limb-girdle muscular dystrophy $2 \mathrm{~J}$ and variations of phenotype. J Neurol Neurosurg Psychiatry 81:1200-1202

23. Blandin G., Marchand S., Charton K., Daniele N., Gicquel E., Boucheteil J. B., Bentaib A., Barrault L., et al., (2013) A human skeletal muscle interactome centered on proteins involved in muscular dystrophies: LGMD interactome. Skelet Muscle. 3, 3-5040-3-3

24. Huovinen S, Penttilä S, Somervuo P, Keto J, Auvinen P, Vihola A, Huovinen S, Pelin K et al (2015) Differential isoform expression and selective muscle involvement in muscular dystrophies. Am J Pathol 185:2833-2842 